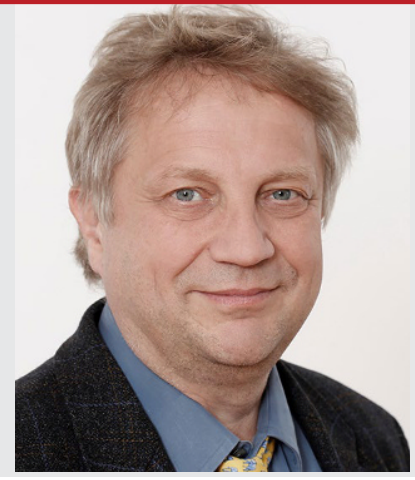

॥ Wir wünschen uns eine angeregte Diskussion!

\author{
Dr. Jürgen Bachmann \\ Schmerzmedizin - Orthopädie - Translationale Medizin \\ Augustastraße 25c, 45525 Hattingen, Deutschland \\ dza@dr-bachmann-hattingen.de
}

\title{
Das kann, muss und soll nicht so bleiben!
}

Liebe LeserInnen und KollegInnen,

kennen Sie den Punkt Magen 36? Was bewirkt eine Stimulation an diesem Ort aus ihrer Sicht? Starke Wirkung im Verdauungssystem, im funktionellen Kernspin bildlich darstellbar? Klinisch hinreichend beforscht? Hat er eine antiinflammatorische Wirkung? Zwar haben wir Ihnen im wissenschaftlichen Teil diesmal keine Original- oder Übersichtsarbeit anzubieten, aber der Beitrag im „Journal Club“ von E. Maronde bringt uns anhand eines hochrangig in Nature 2021 publizierten Artikels einer chinesischen Arbeitsgruppe zu vermeintlich Altbekanntem Details aus dem Blickwinkel der Neuroanatomie: Welche Wirkmechanismen und Wege nimmt die Information von unterhalb des Knies zur Beeinflussung des Magens und welche methodischen Voraussetzungen sind bislang noch nicht hinreichend abgesichert?

Die Verantwortung für unsere Rubrik „Der Kommentar“ ist mit dem Jahreswechsel von Frau C. Tauber-Bachmann auf T. Ots übergegangen. Vorgesehen ist, ausgehend von dem Anspruch der Ganzheitlichkeit und in Erweiterung des Schwerpunktheftes (DZA Heft 3 aus 2020) die globalen Fragen in den Blick zu nehmen, die sich immer mehr, immer vielfältiger und immer drängender stellen. Dies wird komplementär begleitet durch eine Reihe von Rezensionen einschlägiger Literatur: Das Spektrum der Rezensionen geht in diesem Zusammenhang über die Publikationen zur Akupunktur deutlich hinaus.

Die Lösungen der darin angesprochenen Probleme sind nicht offensichtlich, sie liegen nicht vor, sie sind zu erarbeiten. Wir wünschen uns eine angeregte Diskussion - auch wenn der letzte Leserbrief in dieser Fachzeitschrift geraume Zeit zurückliegt: Das muss nicht so bleiben und soll nicht so bleiben.

Ansonsten liegt in diesem Heft der Schwerpunkt im Schwerpunkt: Die Autorengruppe um die Schwerpunktredakteure J. Langhorst und F. Saha beleuchtet umfassend das vielfältig schillernde Thema Fibromyalgiesyndrom: Näheres zu den Themen und Autoren entnehmen Sie bitte dem Editorial zum Schwerpunkt.

A. Wiebrecht hat für diese Ausgabe in bewährter Weise seine Nachrichten aus der Welt der Akupunktur aufbereitet.
Auf der „Anderen Seite“ steht diesmal der Bericht einer Patientin mit multipler Sklerose, die Wege gefunden hat, mit der Erkrankung besser zurechtzukommen, und uns hierzu ein Bild zeigt, das erschauern lässt. Sehen Sie selbst!

Ihr

Jürgen Bachmann

Interessenkonflikt. J. Bachmann gibt an, dass kein Interessenkonflikt besteht. 